\title{
A review of lithium ion batteries electrochemical models for electric vehicles
}

\author{
Jiaping Zhou ${ }^{1}$, Bo Xing ${ }^{1}$, Chunyang Wang ${ }^{1}$ \\ ${ }^{1}$ Beijing CATARC Technologu Co., Ltd., China Automotive Technology \& Research Center, F/9, 2-7 Building ABP, 188Western Rd., \\ South 4th Ring Rd., Fengtai District, Beijing, 100070, China
}

\begin{abstract}
The electrochemical model can reflect the electrochemical reactions inside the lithium ion battery, and the model parameters have practical physical significance. Therefore, it is commonly used in the simulation research of the life prediction and the cell decay mechanism analysis of the lithium ion battery. This paper mainly introduces three commonly used electrochemistry models: P2D model, SP model and extended SP model. The origin and research progress of three kinds of electrochemical models are described. The characteristics of the three models are analyzed and compared. P2D model can describe the battery reactions comprehensively, but the iterative solution is complicated. The SP model simplifies some electrochemical reactions and improves the computational speed of the model, but the accuracy is decreased. In order to solve the shortcoming of SP model, the electrochemical reaction omitted from SP model is introduced again, and the approximate solution is solved by mathematical method, so as to realize the balance between precision and computational complexity of the model. Based on the above analysis and the application scenarios of lithium ion battery, the further development of the lithium ion battery electrochemical model is prospected.
\end{abstract}

\section{Introduction}

The environmental pollution aggravation, global warming, natural resources decreasing and other problems have prompted many countries in the world to conduct a lot of research on vehicles with alternative energy, so as to promote the development of new energy vehicles such as electric vehicles and plug-in hybrid electric vehicles. As the power source of new energy vehicles, the power battery has become the focus of domestic and foreign scholars. Compared with other types of commonly used power batteries, lithium-ion batteries have become a research hotspot due to their advantages such as high specific energy and power, long calendar life and cycle life, low cost, no memory effect, and low pollution[1].

The performance of lithium ion battery is affected by many factors. In order to simulate and predict the lithium ion battery behavior effectively, it is necessary to establish a reasonable model of lithium ion battery. In various lithium-ion battery models, the electrochemical model starts with the internal working process of the battery and gives a reasonable description of the lithium ions behavior in the battery during charging and discharging. All model parameters have clear physical significance. The electrochemical mechanism model can not only describe the macroscopic physical quantities such as voltage and current, but also simulate the important microscopic physical quantities inside the battery[2], which is suitable for application in the degradation analysis and aging analysis of battery performance. There are three types of electrochemical models: P2D model, SP model and extended SP model. In this paper, the principle of lithium ion battery is introduced firstly, and the above three types of electrochemical models are analyzed. Finally, the prospect of lithium ion battery electrochemical model research is made.

\section{Electrochemical model}

\subsection{P2D model}

$\mathrm{J}$ Newman and M Doyle et al proposed as shown in Figure 1 of the lithium ion battery pseudo twodimensional model, this model describes the lithium ion diffusion in the electrolyte, electrode reaction kinetics, ohm's law, charge conservation and material conservation[3]. 


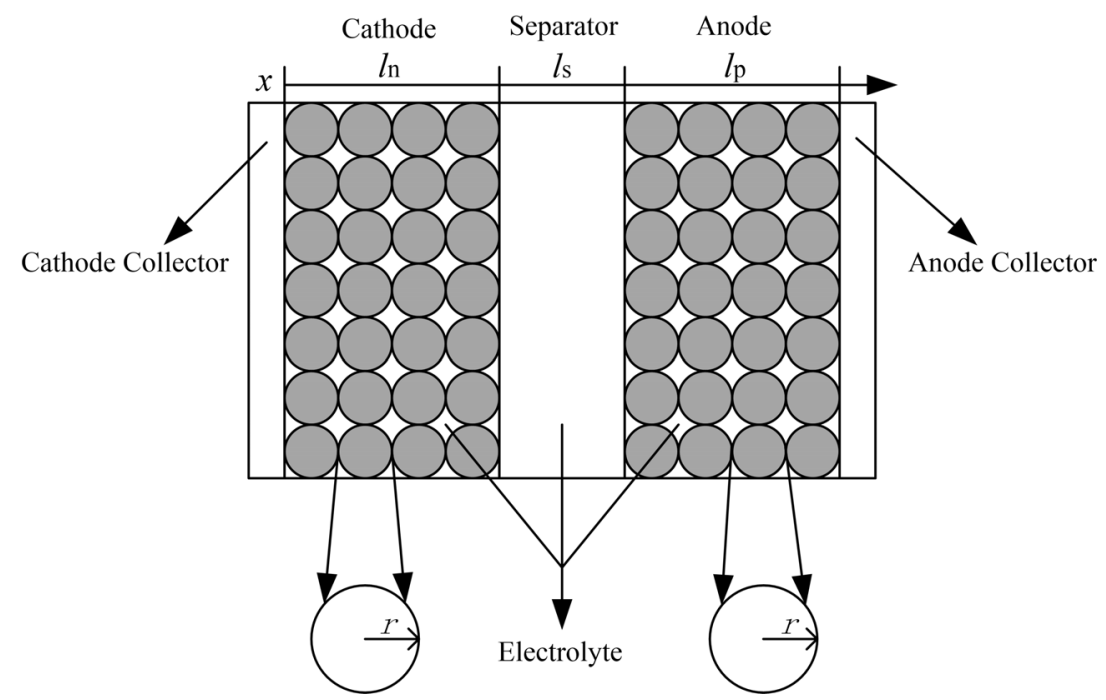

Figure 1. P2D model

P2D model can describe the internal and external characteristics of lithium ion battery at the same time. M Rosas et al. established the P2D model of LiMn2O4 battery and verified the model under dynamic conditions[4]. Zhang Q et al. simulated the charge distribution of $\mathrm{LiCoO} 2$ electrode by P2D model, and semi-empirically treated the symmetric factors in the reaction kinetics equation, and $\mathrm{P} 2 \mathrm{D}$ model was suitable for electrode analysis of $\mathrm{LiCoO} 2$ material[5]. T T Sara et al. proposed a model based on the radius of positive and negative particles and established the P2D model of 18650 LiFePO4 battery[6]. The parameters in P2D model have physical meanings and can reflect the internal state of lithium ion battery during charging and discharging, which is suitable for the microscopic study of lithium ion battery.

\subsection{SP model}

P2D model is composed of partial differential equation and nonlinear algebra, with many iterations, long calculation process and many parameters. The application of P2D model is limited by the difference in computational efficiency and parameter identification. Therefore, many scholars are constantly looking for a reasonable and effective simplification method for P2D model, among which the most widely used is the single particle model. The SP model was proposed by B S Haran et al.[7], the SP model assumes that the concentration of lithium ions in the liquid phase is equal everywhere in the battery and the electric potential of the solid phase is equal everywhere in the electrode. The SP model ignores the uneven distribution of the solid phase diffuse potential of lithium ions in the liquid phase so that the entire electrode can be represented by an active particle.

Based on the P2D model, the SP model introduces the following assumptions, which greatly simplifies the calculation process of the model.

$$
\left\{\begin{array}{l}
j_{\mathrm{n}} \approx \frac{I(t)}{a_{\mathrm{n}} F l_{\mathrm{n}} A_{0}} \\
j_{\mathrm{p}} \approx-\frac{I(t)}{a_{\mathrm{p}} F l_{\mathrm{p}} A_{0}}
\end{array}\right.
$$

Compared with the P2D model, the SP model has fewer solving equations, fewer parameters and higher solving efficiency. Therefore, it has become an electrochemical model that can be applied in real-time systems, and has been applied by some scholars to realtime monitoring of lithium ion concentration in lithium ion batteries[8].

\subsection{Extended SP model}

The accuracy of SP model is reduced due to the oversimplification of electrochemical process, and it is only suitable for the condition of low rate charge and discharge. In order to solve the problems existing in SP model, many scholars have proposed extended SP model. Luo W, Lyu $\mathrm{C}$ et al. introduced the liquid phase diffusion process and the effect of heterogeneous reaction distribution into the SP model and proposed an extended SP model that could charge and discharge at 4C[9]. Han $\mathrm{X}$ and Ouyang $\mathrm{M}$ et al. used a number of first-order processes to approximate the solid-phase diffusion process of lithium ions and treated the concentration distribution of liquid phase in a parabolic fitting way, and proposed an extended SP model that can be applied to BMS[10]. S K Rahimian and S Rayman et al introduced liquid phase potential and matter balance into SP model[11]. I Goto and H Ohkuma et al. introduced the concentration distribution of lithium electrolyte ions into the single-particle model[12]. The electrode is simplified to a single active particle in the extended SP model, and the electrochemical process omitted in the SP model is solved approximately. 


\section{Conclusion}

The electrochemical mechanism models used at this stage are mainly the aforementioned P2D model, SP model and extended SP model. The P2D model proposed by J. Newman and M. Doyle starts with the behavior of lithium ions in the process of charging and discharging describes the behavior of lithium ions in detail. The modeling of the electrochemical mechanism makes the P2D model achieve higher accuracy. The identification of many parameters in P2D is also difficult. Therefore, the P2D model is difficult to apply to engineering.

After the introduction of P2D model, the electrochemical models used by most scholars are simplified based on it by two kinds of simplified ideas: proposing new hypotheses to omit part of the electrochemical process, finding the analytical or approximate solution of each partial differential equation by mathematical method. Either way, the fundamental purpose is to reduce the number of iterative equations.

The SP model omits the partial differential equation of the liquid phase and reduces the electrode to a single particle. The extended SP model still simplifies the electrode into a single active particle but no longer directly solves the partial differential equation by iteration, but uses curve fitting or approximate solution to process the partial differential equation. Although the complexity of the model has increased, the actual computational efficiency has not decreased too much owe to the avoidance of iterative solving.

\section{Prospect}

In this paper, three electrochemical models of lithium ion batteries were introduced and their characteristics are compared. Combined with the application scenario of lithium ion battery, the further research and application of electrochemical model are mainly focused on the following three points.

Based on the electrochemical model, the aging model will be introduced into the electrochemical model. The aging mechanisms of lithium ion batteries in different working processes are analyzed, and the life expectancy of lithium ion batteries will be predicted by combining with the electrochemical model.

In the future, electric vehicles will be further popularized in more regions, and the requirements for lithium ion batteries will be improved. The application scope of the corresponding battery electrochemical model will also be further expanded.

In order to make the electrochemical model applicable in BMS, the electrochemical model should be further improved for the real-time system. The simplification of electrochemical models for real-time systems is the key to the application of electrochemical models in BMS. And it is also one of the research directions of electrochemical models with high accuracy, high real-time performance and high applicable scope in the future lithium ion batteries.

\section{References}

1. Ding, Y., Cano Z P., Yu A. (2019) Automotive LiIon Batteries: Current Status and Future Perspectives. Electrochemical Energy Reviews, 2: 1-28.

2. Zubi, G., Dufo-Lopez, R., Carvalho M. (2018) The lithium-ion battery: State of the art and future perspectives. Renewable and Sustainable Energy Reviews, 89: 292-308.

3. Newman, J., Tiedemann, W. (1975) Porouselectrode theory with battery applications. AIChE Journal, 21: 25-41.

4. Martínez-Rosas, E., Vasquez-Medrano, R., FloresTlacuahuac, A. (2011) Modeling and simulation of lithium-ion batteries. Computers \& Chemical Engineering, 35: 1937-1948.

5. Zhang, Q., Guo, Q., White, R.E. (2007) Semiempirical modeling of charge and discharge profiles for a $\mathrm{LiCoO} 2$ electrode. Journal of Power Sources, 165: 427-435.

6. Sara, T.T., Bernard, M., Lantagne, G. (2018) Modeling and simulation of a commercial graphite$\mathrm{LiFePO} 4$ cell in a full range of C-rates. Journal of Applied Electrochemistry.

7. Haran, B.S., Popov, B.N., White, R.E. (1998) Determination of the hydrogen diffusion coefficient in metal hydrides by impedance spectroscopy. Journal of Power Sources, 75: 56-63.

8. Huang, L., Yao, C. (2016) Working Condition RealTime Monitoring Model of Lithium Ion Batteries Based on Distributed Parameter System and Single Particle Model. Chinese Journal of Chemical Physics, 29: 623-628.

9. Luo, W., Lyu, C., Wang, L. (2013) A new extension of physics-based single particle model for higher charge-discharge rates. Journal of Power Sources, 241: $295-310$

10. Han, X., Ouyang, M., Lu, L. (2015) Simplification of physics-based electrochemical model for lithium ion battery on electric vehicle. Part I: Diffusion simplification and single particle model. Journal of Power Sources, 278: 802-813.

11. Rahimian, S.K., Rayman, S., White, R.E. (2013) Extension of physics-based single particle model for higher charge-discharge rates. Journal of Power Sources, 224: 180-194.

12. Goto, I., Ohkuma, H., Hongo, H. (2016) Feasibility Study of Modified Single-Particle Model for Composite Cathode at High-Rate Discharge. Electrochemistry, 84: 432-437. 